\title{
AN ANSWER TO A.D.WALLACE'S QUESTION ABOUT COUNTABLY COMPACT CANCELLATIVE SEMIGROUPS
}

\author{
DESMOND ROBBIE AND SERGEY SVETLICHNY \\ (Communicated by Franklin D. Tall)
}

\begin{abstract}
It is shown under $\mathrm{CH}$ that there exists a countably compact topological semigroup with two-sided cancellation which is not a topological group. "Wallace's question" of 40 years standing is thus settled in the negative unless $\mathrm{CH}$ is explicitly denied. The example is a topological subsemigroup of an uncountable product of circle groups.
\end{abstract}

\section{INTRODUCTION}

A topological semigroup is a jointly continuous binary operation defined on a Hausdorff topological space and such that the operation is associative. At the annual meeting of the American Mathematical Society in 1953, A.D.Wallace [W] noted that several authors had proved that a compact topological semigroup with two-sided cancellation was a topological group. He further remarked that whether compact might be weakened to countably compact still remained an open question despite contrary published assertions. At the June 1993 Conference on General Topology and Applications at Slippery Rock, USA, renewed interest was expressed in this problem by several speakers including R.W. Heath and D.L.Grant. The problem was also listed by W.W.Comfort in [C1] and [C2], and by R.W.Heath in $[\mathrm{H}]$.

Mukherjea and Tserpes [MT] in 1972 showed that any countably compact cancellative topological semigroup with first countable topology was a topological group. Pfister [P] in 1985, following earlier work of Brand [B], showed that in the completely regular case a countably compact topological semigroup which was algebraically a group was a topological group. In the completely regular case D.L.Grant [G] in 1993 proved that a sequentially compact cancellative topological semigroup was algebraically a group, which coupled with Pfister's result made it a topological group. Later in the paper we show by elementary methods that in a countably compact Frechet space a cancellative topological semigroup is a topological group. There are no restrictions as to regularity. Following a suggestion of J.D.Lawson that attention be focused on countably compact topological subsemigroups of topological groups, a number of interesting properties were noted, including one communicated to us by V.Pestov - each such semigroup is dense in some compact group.

Received by the editors July 19, 1994 .

1991 Mathematics Subject Classification. Primary 22A05, 54D20; Secondary 22A15.

Key words and phrases. Countably compact space, toplogical group, cancellative topological semigroup, Abelian group.

(C)1996 American Mathematical Society 
In the paper all spaces are assumed Hausdorff. As usual an ordinal is the set of smaller ordinals and a cardinal is an initial ordinal. Let $\omega$ and $\omega_{1}$ be the first countable and first uncountable ordinals respectively. Also $\left[\omega_{1}\right]^{\omega}$ denotes the set of all strictly countable subsets of $\omega_{1}$. For a subset $A$ of a group $G$ we denote by $\langle A\rangle$ the semigroup algebraically generated by $A$ and by $[A]$ the topological closure of $A$ in $G$. A countably (sequentially) compact space is one in which each infinite subset (sequence) has a limit point (a convergent subsequence). A Frechet space is a space in which each limit point of a subset is the limit point of some sequence in the subset.

The main purpose of this paper is to prove the following:

Theorem A $(\mathrm{CH})$. There is an example of an Abelian completely regular countably compact topological semigroup with two-sided cancellation which is not a group.

The following result is well known.

Lemma A.1 $(\mathrm{CH})$. It is possible to enumerate $\left[\omega_{1}\right]^{\omega}$ as $\left\{I_{\alpha}: \omega \leqslant \alpha<\omega_{1}\right\}$ in such a way that $I_{\alpha} \subseteq \alpha$ for each $\omega \leqslant \alpha<\omega_{1}$.

We also call on a paper of Tkachenko [TK, Theorem 1 and Note 2], in which, under $\mathrm{CH}$, he shows the existence, as a topological subgroup of an uncountable product of circle groups, of a group $G$ with the following properties: $G$ is (noncompact) countably compact, hereditarily separable, hereditarily normal, connected and locally connected; $G$ is algebraically isomorphic to the free Abelian group of cardinality $\omega_{1}$; and the closure of any infinite subset of $G$ is uncountable. Thus we have the following

Lemma A.2 (CH). There exists a (non-compact) countably compact subgroup $G$ of an uncountable product of circle groups, such that $G$ is torsion free and the closure of any infinite subset of $G$ is uncountable.

Lemma A.3. Let $G$ be an Abelian, torsion free, countably compact group such that the closure of any infinite subset of $G$ is uncountable, and let e denote the identity of $G$. Suppose that $T$ is any countably infinite subsemigroup of $G$ such that $e \notin T$. We denote by $T^{-1}$ the set of inverses of elements of $T$. Let $B$ be any infinite subset of $T$. Then there exists $g$, a limit point of $B$, such that for each $n \in \omega, g^{n} \notin\left\langle T \cup T^{-1}\right\rangle$ and, moreover, $e \notin\langle T \cup\{g\}\rangle$.

Proof. First of all let us note that the set $[B]$, the closure of $B$ in $G$, is uncountable. As $T$ is countably infinite so is $T^{-1}$. We note that for each $n \in \omega$ the map $p_{n}: G \rightarrow G$, by $p_{n}(x)=x^{n}$, is one-to-one. Indeed, if $x_{1}^{k}=x_{2}^{k}$, then $\left(x_{1} x_{2}^{-1}\right)^{k}=e$, and as $G$ is torsion free, $x_{1} x_{2}^{-1}=e$, so $x_{1}=x_{2}$. Now as $T, T^{-1}$ are each countable so is the group $\left\langle T \cup T^{-1}\right\rangle$. Thus for each $n \in \omega$ the set $p_{n}^{-1}\left(\left\langle T \cup T^{-1}\right\rangle\right)$ is countable. So

$$
A=\bigcup\left\{p_{n}^{-1}\left(\left\langle T \cup T^{-1}\right\rangle\right): n \in \omega\right\}
$$

is also countable. Since $[B]$ is uncountable, we may choose $g \in[B]$ such that $g \notin A$. Then for each $n \in \omega, g^{n} \notin\left\langle T \cup T^{-1}\right\rangle$, and $e \notin\langle T \cup\{g\}\rangle$.

Proof of Theorem A. From Lemma A.2, under CH there exists a countably compact torsion free Abelian group $G$ such that the closure of any countably infinite subset of $G$ is uncountable. Again $e$ denotes the identity of $G$. Let $a \in G, a \neq e$. The generated subsemigroup $\langle a\rangle=\left\{a^{n}: n \in \omega\right\}$ is countably infinite and $e \notin\langle a\rangle$, since 
$G$ is torsion free. Next from the enumeration given in Lemma A.1 we have $I_{\omega} \subseteq \omega$. Thus $B=\left\{a^{n}: n \in I_{\omega}\right\}$ must be infinite, since $I_{\omega}$ is countably infinite. Apply Lemma A.3 to the sets $B$ and $T=\langle a\rangle$ to find $a_{\omega}$ a limit point of $B$, such that for each $n \in \omega, a_{\omega}^{n} \notin\left\langle T \cup T^{-1}\right\rangle$, and $e \notin\left\langle T \cup\left\{a_{\omega}\right\}\right\rangle$. Let

$$
S_{\omega}=\left\langle T \cup\left\{a_{\omega}\right\}\right\rangle=\left\{a^{n}: n \in \omega\right\} \cup\left\{a_{\alpha}: \omega \leqslant \alpha<\omega+\omega\right\} .
$$

Note that for $\alpha_{1} \neq \alpha_{2}$ it follows that $a_{\alpha_{1}} \neq a_{\alpha_{2}}$.

We construct by transfinite induction for each $\lambda, \omega \leqslant \lambda<\omega_{1}$ sets $S_{\lambda}$, and ordinals $\tau(\lambda)<\omega_{1}$ such that

(1) $S_{\lambda}=\left\{a^{n}: n \in \omega\right\} \cup\left\{a_{\alpha}: \omega \leqslant \alpha<\tau(\lambda)\right\}$ and if $\alpha_{1} \neq \alpha_{2}$, then $a_{\alpha_{1}} \neq a_{\alpha_{2}}$.

(2) $S_{\lambda}$ is a subsemigroup of $G$ and $e \notin S_{\lambda}$.

(3) $\lambda \leqslant \tau(\lambda)<\omega_{1}$.

(4) If $\omega \leqslant \lambda^{\prime} \leqslant \lambda^{\prime \prime} \leqslant \lambda$, then $S_{\lambda^{\prime}} \subseteq S_{\lambda^{\prime \prime}} \subseteq S_{\lambda}$.

(5) If $\alpha<\lambda$, then $a_{\alpha} \in S_{\alpha}$.

(6) $\left(\left\{a^{n}: n \in I_{\lambda}\right\} \cup\left\{a_{\alpha}: \omega \leqslant \alpha, \alpha \in I_{\lambda}\right\}\right) \subseteq S_{\lambda}$.

Conditions (5) and (6) follow from Lemma A.1 together with conditions (1) and (3) and are used in the sequel.

The case $\lambda=\omega$ is established above and $\tau(\omega)=\omega+\omega$. For $\lambda>\omega$ there are two options: either $\lambda$ is limit or non-limit.

(a) $\lambda$ is not a limit ordinal. Apply condition (6) to the set $S_{\lambda-1}$ to get

$$
B=\left(\left\{a^{n}: n \in I_{\lambda-1}\right\} \cup\left\{a_{\alpha}: \omega \leqslant \alpha, \alpha \in I_{\lambda-1}\right\}\right) \subseteq S_{\lambda-1} .
$$

Also by condition (1) and the fact that $G$ is torsion free, $B$ is an infinite subset of $S_{\lambda-1}$. By Lemma A.3 and condition (2) we may choose $a_{\tau(\lambda-1)+1}$ a limit point of $B$ such that for each $n \in \omega, a_{\tau(\lambda-1)+1}^{n} \notin\left\langle S_{\lambda-1} \cup S_{\lambda-1}^{-1}\right\rangle$ and $e \notin\left\langle S_{\lambda-1} \cup\left\{a_{\tau(\lambda-1)+1}\right\}\right\rangle$.

Put $\tau(\lambda)=\tau(\lambda-1)+\omega$ and

$$
\begin{aligned}
S_{\lambda} & =\left\langle S_{\lambda-1} \cup\left\{a_{\tau(\lambda-1)+1}\right\}\right\rangle \\
& =S_{\lambda-1} \cup\left\{a_{\alpha}: \tau(\lambda-1)+1 \leqslant \alpha<\tau(\lambda-1)+w\right\} \\
& =\left\{a^{n}: n \in \omega\right\} \cup\left\{a_{\alpha}: \omega \leqslant \alpha<\tau(\lambda)\right\} .
\end{aligned}
$$

The choice of the point $a_{\tau(\lambda-1)+1}$ ensures that $S_{\lambda}$ satisfies condition (1). This is because, if $s_{1}\left(a_{\tau(\lambda-1)+1}\right)^{n_{1}}=s_{2}\left(a_{\tau(\lambda-1)+1}\right)^{n_{2}}$ for some $s_{1}, s_{2} \in S_{\lambda-1}$ and $n_{1} \leqslant n_{2}$, then $\left(a_{\tau(\lambda-1)+1}\right)^{n_{2}-n_{1}} \in\left\langle S_{\lambda-1} \cup S_{\lambda-1}^{-1}\right\rangle$ which has been excluded. Condition (2) is also immediate. To check (3) we notice that $\lambda-1 \leqslant \tau(\lambda-1)$ which holds by condition (3) for $\lambda-1$, and so

$$
\lambda \leqslant \tau(\lambda-1)+1 \leqslant \tau(\lambda-1)+\omega=\tau(\lambda) .
$$

To see that (4) is satisfied we can use $S_{\lambda-1} \subseteq S_{\lambda}$ and it remains to apply induction assumptions for $S_{\lambda-1}$. It is pointed out above that conditions (5) and (6) follow directly from (1) - (4) and Lemma A.1, so they are satisfied automatically.

(b) $\lambda$ is a limit ordinal. In this case first define the set $S_{\lambda}^{*}$ and the ordinal $\tau^{*}(\lambda)$ as follows:

$$
\begin{aligned}
S_{\lambda}^{*} & =\bigcup\left\{S_{\alpha}: \alpha<\lambda\right\}, \\
\tau^{*}(\lambda) & =\bigcup\{\tau(\alpha): \alpha<\lambda\} .
\end{aligned}
$$


Clearly $S_{\lambda}^{*}$ is a subsemigroup of $G$ by conditions (2) and (4), is countable, and $e \notin S_{\lambda}^{*}$. Let

$$
B=\left\{a^{n}: n \in I_{\lambda}\right\} \cup\left\{a_{\alpha}: \omega \leqslant \alpha, \alpha \in I_{\lambda}\right\} .
$$

Since $I_{\lambda} \subseteq \lambda$, then for each pair $\alpha_{1}, \alpha_{2} \in I_{\lambda}$ it follows that $\alpha_{1}, \alpha_{2}<\lambda$. So if $\alpha_{1}<\alpha_{2}$, then by conditions (4) and (5) applied we have that $a_{\alpha_{1}}, a_{\alpha_{2}} \in S_{\alpha_{2}}$. Hence by condition (1), $a_{\alpha_{1}} \neq a_{\alpha_{2}}$. Also the group $G$ is torsion free. So $B$ is an infinite set. Since $I_{\lambda} \subseteq \lambda$ and, by induction assumption (3), $\lambda \leqslant \bigcup\{\tau(\alpha): \alpha<\lambda\}=\tau^{*}(\lambda)$, we get

$$
\begin{aligned}
B & \subseteq\left\{a^{n}: n \in I_{\lambda}\right\} \cup\left\{a_{\alpha}: \omega \leqslant \alpha<\lambda\right\} \\
& \subseteq\left\{a^{n}: n \in I_{\lambda}\right\} \cup\left\{a_{\alpha}: \omega \leqslant \alpha<\tau^{*}(\lambda)\right\} \\
& =\bigcup\left\{S_{\alpha}: \alpha<\lambda\right\} \\
& =S_{\lambda}^{*} .
\end{aligned}
$$

Apply Lemma A.3 to the sets $B$ and $S_{\lambda}^{*}$ to get a limit point $a_{\tau^{*}(\lambda)+1}$ of the set $B$ such that for each $n \in \omega, a_{\tau^{*}(\lambda)+1}^{n} \notin\left\langle S_{\lambda}^{*} \cup S_{\lambda}^{*-1}\right\rangle$ and $e \notin\left\langle S_{\lambda}^{*} \cup\left\{a_{\tau^{*}(\lambda)+1}\right\}\right\rangle$.

Define $\tau(\lambda)=\tau^{*}(\lambda)+\omega$ and

$$
\begin{aligned}
S_{\lambda} & =\left\langle S_{\lambda}^{*} \cup\left\{a_{\tau^{*}(\lambda)+1}\right\}\right\rangle \\
& =S_{\lambda}^{*} \cup\left\{a_{\alpha}: \tau^{*}(\lambda)+1 \leqslant \alpha<\tau^{*}(\lambda)+\omega\right\} \\
& =\left\{a^{n}: n \in \omega\right\} \cup\left\{a_{\alpha}: \omega \leqslant \alpha<\tau(\lambda)\right\} .
\end{aligned}
$$

We now check that (1) - (4) are satisfied for the constructed sets $S_{\lambda}$ and $\tau(\lambda)$. If $\alpha_{1} \neq \alpha_{2}$, then $a_{\alpha_{1}} \neq a_{\alpha_{2}}$ as above in the non-limit case. Next, as shown above and from the definition of $\tau(\lambda)$

$$
\lambda \leqslant \bigcup\{\tau(\alpha): \alpha<\lambda\}=\tau^{*}(\lambda) \leqslant \tau(\lambda) .
$$

So (3) holds. Condition (2) is immediate and (4) is a routine exercise.

Now, let

$$
S=\bigcup\left\{S_{\lambda}: \omega \leqslant \lambda<\omega_{1}\right\} .
$$

First, $S$ is an Abelian, completely regular, cancellative topological semigroup as a subsemigroup of the Abelian topological group $G$ (by conditions (2) and (4)). Further, $S$ is not a group for $e \notin S$ by condition (2). Finally, $S$ is countably compact. Indeed, from our construction

$$
S=\left\{a^{n}: n \in \omega\right\} \cup\left\{a_{\alpha}: \omega \leqslant \alpha<\omega_{1}\right\}
$$

and therefore, for any countable subset $A \subseteq S$, there exists $\lambda<\omega_{1}$ such that

$$
A=\left\{a^{n}: n \in I_{\lambda}\right\} \cup\left\{a_{\alpha}: \omega \leqslant \alpha, \alpha \in I_{\lambda}\right\} .
$$

Hence, by condition (6), $A \subseteq S_{\lambda}$ and $A$ either has the limit point $a_{\tau(\lambda-1)+1} \in S_{\lambda} \subseteq$ $S$, if $\lambda$ is not a limit ordinal, or $a_{\tau^{*}(\lambda)+1} \in S_{\lambda} \subseteq S$ otherwise.

Remark 1. In a compact semigroup there is always at least one idempotent element. We have shown under $\mathrm{CH}$ that even in the completely regular cancellative case there is a countably compact semigroup with no idempotent element. 
Remark 2. It follows from comments preceding Lemma 2 that the semigroup constructed in Theorem A is hereditarily separable and so is of countable tightness. Under $\mathrm{CH}$ this answers in the negative Question 1 from $[\mathrm{G}]$.

Remark 3. Lemma A.1 may be modified (see for example van Douwen [D]) so as to give in ZFC a sufficient enumeration of the countably infinite subsets of the continuum ordinal to enable changes mutatus mutandis to our construction so that Theorem A holds in ZFC, provided only that one can find in ZFC a torsion free Abelian countably compact group in which each infinite subset has closure of cardinality greater than or equal to the continuum.

So far the authors have been unable to construct such a group, even though it would not need to have the additional properties of Tkachenko's group under $\mathrm{CH}$. However, following circulation of the present results in preprint form at a talk given at the August, 1994 Amsterdam conference on Topology and Applications, Tomita [TO] has reported an improvement by construction under MA of a group with the desired properties.

Theorem B. Let $S$ be a cancellative topological semigroup defined on a countably compact Frechet space. Then $S$ must be a topological group.

There are no additional assumptions regarding regularity of the topology of $S$. Our first lemma assumes only that our space $S$ is sequentially compact.

Lemma B.1. If $S$ is any sequentially compact cancellative topological semigroup, then $S$ has a unique idempotent which is a two-sided identity $\mathbf{1}$ for $S$. Moreover $S$ is algebraically a group and hence the topology is homogeneous.

Proof. Here we use some modifications of ideas from the paper [GKO]. It is shown first that there exist $x, y \in S$ such that $x y=y$.

Consider any $x \in S$ and $\left\{x^{n}: n \in \omega\right\} \subseteq S$. If $x^{n}=x^{m}$ for some $n>m$, then $x^{n-m} x^{m}=x^{m}$. Consider instead that $\left\{x^{n}: n \in \omega\right\}$ is infinite. Take some convergent subsequence $\left\{x^{n(k)}: k \in \omega\right\} \rightarrow z_{0}$. Let us consider $\left\{x^{n(k)-1}: k \in\right.$ $\omega, n(k)>1\}$. Again there is some convergent subsequence $\left\{x^{n(k(m))-1}: m \in\right.$ $\omega\} \rightarrow z_{1}$. As multiplication is continuous, $x z_{1}=z_{0}$. Continuing this process we get $\left\{z_{n}: n \in \omega\right\}$ such that $x^{n} z_{n}=z_{0}$. If $z_{n}=z_{m}$, then $z_{0}=x^{n} z_{n}=x^{m} z_{m}$ implies that $x^{n}=x^{m}$. So $\left\{z_{n}: n \in \omega\right\}$ is infinite. Also $\left\{z_{n(k)}: k \in \omega\right\}$ is infinite so there exists a subsequence $\left\{z_{n(k(l))}: l \in \omega\right\}$ converging to some point $z^{\prime} \in S$. As $x^{n(k(l))} z_{n(k(l))}=z_{0}$, then $\left\{x^{n(k(l))} z_{n(k(l))}: l \in \omega\right\} \rightarrow z_{0}$. On the other hand, $\left\{x^{n(k(l))}: l \in \omega\right\} \rightarrow z_{0}$ and $\left\{z_{n(k(l))}: l \in \omega\right\} \rightarrow z^{\prime}$. Therefore $\left\{x^{n(k(l))} z_{n(k(l))}: l \in \omega\right\} \rightarrow z_{0} z^{\prime}$ and so $z_{0} z^{\prime}=z_{0}$.

That there is a unique idempotent which is an identity $\mathbf{1}$ for $S$ now follows by elementary algebra. Moreover, as translation by any element of $S$ is a continuous function, $S x$ and $x S$ are sequentially compact. So, $S, x S$ and $S x$ all have the same identity 1. Now $S \subseteq S \mathbf{1} \subseteq S(S x) \subseteq S^{2} x \subseteq S x \subseteq S$. So $S=S x$. Similarly, $S=x S$. Thus $S$ is algebraically a group. The topology of $S$ is then homogeneous.

Lemma B.2. If $S$ is a topological semigroup which is algebraically a group and which has a countably compact Frechet topology, then $S$ is a topological group.

Proof. As the topology of $S$ is homogeneous we need only prove the continuity of the inverse operation at $\mathbf{1}$. Suppose that the inverse operation is not continuous at 
1. Then there exists a neighborhood $U(\mathbf{1})$ such that for any neighborhood $V(\mathbf{1})$, $(V(\mathbf{1}))^{-1}$ is not contained in $U(\mathbf{1})$. Thus there is a set $A \subseteq S$ which has $\mathbf{1}$ as a limit point and $A^{-1}=\left\{a^{-1}: a \in A\right\}$ does not intersect $U(\mathbf{1})$. As we have a Frechet space there exists $\left\{a_{n}: n \in \omega\right\} \subseteq A$ such that $\left\{a_{n}: n \in \omega\right\} \rightarrow \mathbf{1}$. Then by countable compactness the set $\left\{a_{n}^{-1}: n \in \omega\right\}$ has a limit point $q$ such that $q \notin U(\mathbf{1})$. Again as $S$ is a Frechet space there exists $\left\{b_{k}^{-1}: k \in \omega\right\} \subseteq\left\{a_{n}^{-1}: n \in \omega\right\}$ such that $\left\{b_{k}^{-1}: k \in \omega\right\} \rightarrow q$. Clearly $\left\{b_{k}^{-1}: k \in \omega\right\}$ must have arbitrarily high members of $\left\{a_{n}^{-1}: n \in \omega\right\}$ in it so we may choose a subsequence of $\left\{b_{k}^{-1}: k \in \omega\right\}$, say $\left\{b_{k(l)}^{-1}: l \in \omega\right\}$, which defines a subsequence $\left\{a_{n(l)}^{-1}: l \in \omega\right\}$ of $\left\{a_{n}^{-1}: n \in \omega\right\}$ with $\left\{a_{n(l)}^{-1}: l \in \omega\right\} \rightarrow q$. Also $\left\{a_{n(l)}: l \in \omega\right\} \rightarrow \mathbf{1}$. So $\left\{a_{n(l)} a_{n(l)}^{-1}: l \in \omega\right\} \rightarrow \mathbf{1} q=q$. On the other hand, $\left\{\mathbf{1}=a_{n(l)} a_{n(l)}^{-1}: l \in \omega\right\} \rightarrow \mathbf{1}$. Therefore $\mathbf{1}=q$. This contradicts $q \notin U(\mathbf{1})$.

Question 1. Is there a sequentially compact topological semigroup (not assumed to be regular) which is algebraically a group but is not a topological group ?

We are grateful to the referee for suggesting the following question.

Question 2. Is there a cardinal $\kappa$ with the property that any $\kappa$-compact cancellative topological semigroup is a topological group? [By $\kappa$-compact is meant a space in which every open cover of cardinality less than $\kappa$ has a finite subcover.]

\section{REFERENCES}

[B] N.Brand, Another note on the continuity of the inverse, Arch. Math. 39 (1982), 241-245. MR 84b:22001

[C1] W. W. Comfort, Topological groups, Handbook of set-theoretic topology, North-Holland, Amsterdam, 1984, pp. 1143-1263. MR 86g:22001

[C2] W. W. Comfort, Problems on topological groups and other homogeneous spaces, Open problems in topology, North-Holland, Amsterdam, 1990, pp. 313-347. CMP 91:03

[D] Eric K. van Douwen, The product of two countably compact topological groups, Trans. Amer. Math. Soc. 262 (1980), 417-427. MR 82b:22002

[GKO] B.Gelbaum, G.K.Kalisch and J.M.H.Olmsted, On the embedding of topological semigroups and integral domains, Proc. Amer. Math. Soc. 2 (1951), 807-821. MR 13:206f

[G] D. L. Grant, Sequentially compact cancellative topological semigroups : some progress on the Wallace problem, in "Papers on General Topology and Applications, Seventh Summer Conference at the University of Wisconsin" (Madison, 1991), Annals of the New York Acad. Sci. 704 (1993), Susan Andima et al (eds.), NY, 150-154. MR 95b:22006

[H] R. W. Heath, Some nonmetric, first countable, cancellative topological semigroups that are generalized metric spaces, Topology and its Appl. 44 (1992), 167-173. MR 93h:22003

[MT] A.Mukherjea and N.A.Tserpes, A note on countably compact semigroups, J. Austral. Math. Soc. 13 (1972), 180-184. MR 46:3680

[P] H.Pfister, Continuity of the inverse, Proc. Amer. Math. Soc. 95 (1986), 312-314. MR 87a:22004

[TK] M. G. Tkachenko, Countably compact and pseudocompact topologies on free abelian groups, Izvestiya V. U. Z., Matematika 34 (1990), 68-75. MR 92e:54044

[TO] Artur H. Tomita, Extending the Robbie-Svetlichny solution of Wallace's problem to MA, preprint (November 1994).

[W] A.D. Wallace, The structure of topological semigroups, Amer. Math. Soc. Bull. 61 (1955), 95-112. MR 16:796d

Department of Mathematics, The University of Melbourne, Parkville, Victoria, Australia, 3052

E-mail address: robbie@mundoe.maths.mu.oz.au

E-mail address: svet@mundoe.maths.mu.oz.au 